Infect Dis |4(I):70-72 (2008); doi:I0.320I/ eid I 401.070704 .

28. Rosenblatt-Farrell N. The landscape of antibiotic resistance. Environ Health Perspect I I7(6):A244-A250 (2009); doi:I0.1289/ehp. I 7 a244.

29. Aarestrup FM, ed.Antimicrobial Resistance in Bacteria of Animal Origin. Washington, DC:ASM Press (2006).

30.Allen HK, et al. Call of the wild: antibiotic resistance genes in natural environments. Nature Rev Microbiol 8(4):25I-259 (20I0); doi: I0.1038/ nrmicro2312.

31.Yim G, et al. The truth about antibiotics. Int J Med Microbiol 296(2-3)।63-I70 (2006); doi:10.1016/j.jijmm.2006.01.039.

32. Silbergeld EK, et al. Industrial food animal production, antimicrobial resistance, and human health. Annu Rev Public Health 29:15I-I69 (2008); doi:I0.I I 46/annurev. publhealth.29.020907.090904

33. Munir M, Xagoraraki I. Levels of antibiotic resistance genes in manure, biosolids, and fertilized soil.J Environ Qual 40(I):248-255 (20II); doi:10.2I34/jeq2010.0209.

34. Las bacterias gram negativas, cuyas membranas son más delgadas, tienden más a desarrollar resistencia que las bacterias gram positivas, de membranas más gruesas. 35. AstraZeneca. Dedicated TB Research [website]. Södertälje, Sweden:AstraZeneca
Global (20II). Disponible en: http://tinyurl. com/679gx84 [consultado el 6 de abril de 20I I]. 36. AstraZeneca. Bacterial Resistance to Antibiotics is a Global Health Threat. Creative Collaboration is the Key to Successfully Meeting the Challenge [página web]. Södertälje, Sweden:AstraZeneca Global (updated I Nov 2010). Disponible en: http://tinyurl.com/3hr9tp4 [consultado el 6 de abril de 20II].

37. Resources for the Future Launches Global Antibiotic Resistance Initiative [press release]. Seattle,WA: Fundación Bill y Melinda Gates (7 de enero de 2009). Disponible en: http://tinyurl. com/6crood4 [consultado el 6 de abril de $20 \mathrm{I}$ ]]. 38. ECDC. Trans Atlantic Task Force on Antimicrobial Resistance-TATFAR [website].

Estocolmo, Suecia: Centro Europeo de Prevención y Control de Enfermedades (200520II). Disponible en: http://tinyurl.com/5sdxlpm [consultado el 6 de abril de 20II].

39. Swedish Presidency of the European Union [website]. 2009 EU-U.S. Summit Declaration. Estocolmo, Suecia: Secretaría de Comunicaciones de la Presidencia de la Unión Europea de Suecia, Oficina del Primer Ministro de Suecia (2009). Disponible en: http://tinyurl.com/3u9ndv5

[consultado el 6 de abril de 201 I].

40. ECDC. European Antibiotic Awareness Day [website]. Estocolmo, Suecia: Centro Europeo de Prevención y Control de Enfermedades (2005-

20II). Disponible en: http://tinyurl.com/5to66ko [consultado el 6 de abril de 201 I] 41. ReAct.Antibiotic Awareness Day-Not Only in Europe [press release]. Uppsala, Suecia:ReAct, Universidad de Uppsala (22 de diciembre de 2010). Disponible en: http://tinyurl.com/6zmjau9 [consultado el 6 de abril de 20I I]. 42. ECDC. ECDC Marks World Health Day 201 I with Situation Update on Antimicrobial Resistance in Europe [press release]. Estocolmo, Suecia: Centro Europeo de Prevención y Control de Enfermedades (19 de abril de 20I I). Disponible en: http://tinyurl.com/ykpjtlz [consultado el 19 de abril de 201 I].

43. Kumarasamy KK, et al. Emergence of a new antibiotic resistance mechanism in India, Pakistan, and the UK: a molecular, biological, and epidemiological study. Lancet Infect Dis I0(9):597-602 (2010); doi: I0.10I6/SI4733099(I0)70143-2.

44.Walsh TR, et al. Dissemination of NDM-I positive bacteria in the New Delhi environment and its implications for human health: an environmental point prevalence study. Lancet Infect Dis; doi:10.1016/SI473-3099(II)70059-7 [publicado en línea el 7 de abril de 20II]. 45. Aarestrup FM, et al. Changes in the use of antimicrobials and the effects on productivity of swine farms in Denmark.J Am Vet Med Assoc 7I(7):726-733 (20I0); doi:I0.2460/ajvr.7I.7.726.

El fracaso no es fatal, pero la incapacidad de cambiar puede serlo. John R. Wooden, entrenador de baloncesto (1910-2010)

\section{El tabaquismo y el humo de segunda mano Estimación global de la carga de HSM*}

$\mathrm{N}$ o importa cómo se le llame en las diversas partes del mundo - tabaquismo pasivo, humo ambiental de tabaco o humo de segunda mano (HSM)-, la exposición a las emisiones de humo de tabaco de los fumadores fue causa de muerte prematura de aproximadamente 603,000 personas en el año 2004, según un equipo de investigadores académicos y de la Organización Mundial de la Salud
(OMS). ${ }^{1}$ Mattias Öberg, del Instituto Karolinska, dirigió la primera evaluación global de la carga de HSM, que fue patrocinada por el Consejo Nacional de Salud y Bienestar de Suecia y Filantropías Bloomberg.

A mediados de los 1980 se confirmó por primera vez que el HSM tiene efectos adversos sobre la salud. ${ }^{2}$ Actualmente, entre los efectos asociados se incluyen enfermedades del corazón, cáncer de pulmón, empeoramiento del asma, síndrome de muerte súbita infantil, etc. Sin embargo, hasta ahora no se han recopilado en

*Publicado originalmente en Environmental Health Perspectives, volumen 119, número 2, febrero de 2011, página A66 
gran escala datos sobre las muertes y enfermedades de los no fumadores.

"Se han hecho antes cálculos del impacto del HSM en países específi$\cos ^{\prime \prime}$, señala el epidemiólogo Jonathan Samet, de la Universidad del Sur de California, quien no participó en el nuevo estudio. "El nuevo artículo de Öberg y sus colegas es importante porque presenta un panorama global."

Los investigadores buscaron datos confiables sobre el hábito de fumar por sexo y edad en la literatura científica, en los reportes sobre salud pública y en las bases de datos gubernamentales. En los casos en que no existían los datos necesarios, creaban modelos para extrapolar de regiones bien estudiadas a países con poca disponibilidad de datos. Una importante fuente de datos sobre la exposición de los niños al humo de tabaco fue la Encuesta Global de Tabaquismo Juvenil, ${ }^{3}$ copatrocinada por los Centros para el Control y Prevención de Enfermedades de Estados Unidos en más de 120 países. Esta encuesta escolar se administra anualmente a niños y adolescentes de 13 a 15 años de edad para evaluar el uso de productos de tabaco y exposición al HSM en los niños.

Öberg señala que el equipo adoptó un enfoque conservador en su evaluación. Por ejemplo, los investigadores optaron por no incluir muertes o enfermedades para las cuales no hubiera evidencias claras y contundentes de una relación causal con la exposición al HSM (una de estas enfermedades es el derrame cerebral). Excluyeron aquellas enfermedades que han sido vinculadas causalmente al HSM cuando no se contaba con estadísticas internacionales de salud comparables (un ejemplo fue el del síndrome de muerte infantil súbita). El equipo tampoco incluyó el cáncer de mama premenopáusico, puesto que la relación entre esta enfermedad y el HSM sigue suscitando controversias en la comunidad científica. ${ }^{4}$

"Es difícil encontrar datos verdaderamente exactos [para algunos de estos resultados]," dice Öberg. Es más, añade, algunos de los efectos apenas pueden relacionarse directamente con la exposición al HSM. Por ejemplo, a pesar de que hay evidencias de peso que sugieren vínculos entre el derrame cerebral, la enfermedad pulmonar obstructiva crónica (EPOC) y el HSM, no se han confirmado estos vínculos mediante metaanálisis epidemiológicos convincentes. ${ }^{1}$

También ha sido difícil encontrar datos sobre la exposición al HSM en revisiones por pares de alta calidad; se cuenta con muchos más datos sobre el número de fumadores activos, señala Öberg. Añade que, si bien es probable

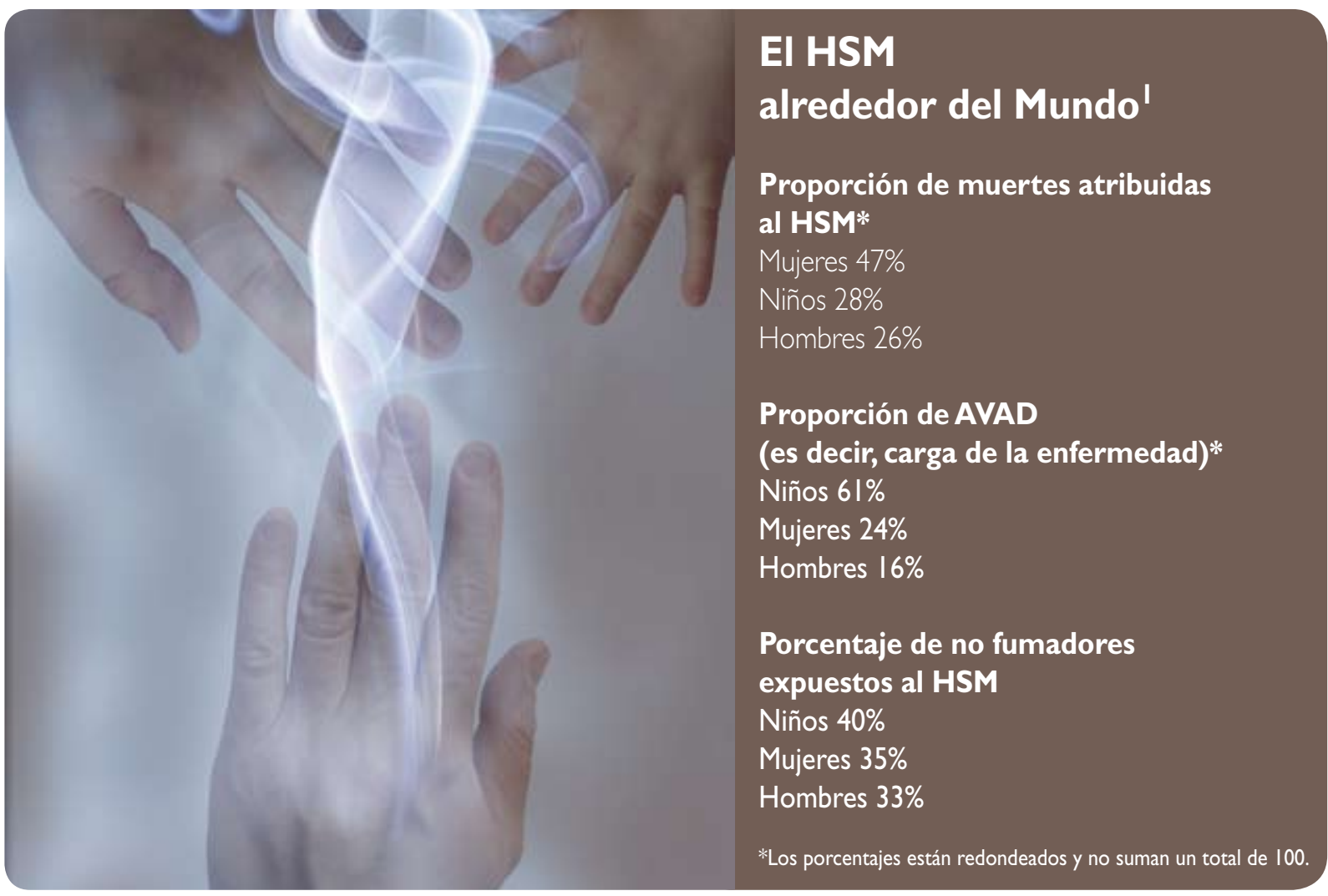


que los fumadores se vean afectados por el tabaquismo pasivo, no se los incluyó en la evaluación principal realizada por el equipo. Si se los hubiera incluido, el índice de mortalidad estimado se habría incrementado en un 30\% aproximadamente; se incluyó a ex fumadores; sin ellos, el número total de muertes habría disminuido en un 17 por ciento. ${ }^{1}$

Al final, el equipo estimó que la proporción global de personas expuestas al HSM en diversos entornos en el año 2004 fue del $40 \%$ del total de los niños (definidos como de entre 0 y 14 años de edad), el 33\% de los hombres no fumadores y el $35 \%$ de las mujeres no fumadoras en todo el mundo. Pero esas proporciones variaban en las diversas regiones según los hábitos de fumar, según si la población era urbana o rural, según las regulaciones del país y según otros factores. Por ejemplo, se estimó que en la región que incluye a Bielorrusia, Estonia, Hungría, Kazajistán, Letonia, Lituania, la República de Moldavia, la Federación Rusa y Ucrania, alrededor de dos tercios de los fumadores de todos los grupos de edad y sexo se hallaban expuestos. En el sur y el noreste de África se estimó que sólo $12 \%$ de los niños estaban expuestos, y el porcentaje de hombres y mujeres expuestos era aun menor. ${ }^{1}$

La carga de morbilidad por exposición al HSM, medida en años de vida ajustados por discapacidad (AVAD), también variaba según la región, y se estimó que era más elevada en los países de bajos ingresos en el sureste de Asia y en el este del Mediterráneo, en comparación con Europa. En la mayoría de los adultos las enfermedades eran sobre todo asma e isquemia del corazón. ${ }^{1}$

Lo que más llama la atención es que los niños de 5 años de edad resultaron ser quienes más padecen infecciones respiratorias en los países más pobres, donde la desnutrición y la falta de atención médica adecuada

No importa qué supuestos se utilicen, el impacto [del HSM] sobre la salud de niños y adultos sigue siendo de gran importancia para la salud pública.

Bart Ostro

Centro de Investigación

de Epidemiología Ambiental

puede conducir a índices de morbilidad y mortalidad más elevados en niños con otros problemas de salud que se exacerban con la exposición al HSM. El equipo calculó que en general los niños padecen $61 \%$ de la carga total de enfermedades causadas por el HSM. ${ }^{1}$

"Los niños siguen estando expuestos en casa", aun en aquellos países que cuentan con leyes que prohíben fumar en lugares públicos, dice Heather Wipfli, experta en políticas de la Universidad de California del Sur y coautora, junto con Samet, de un comentario sobre las nuevas investigaciones publicado en The Lancet. $^{5}$ Sin embargo, Wipfli considera que la exposición al HSM es en gran medida un problema de las mujeres: únicamente $10 \%$ de las mujeres de todo el mundo fuma, explica, pero de los 603000 no fumadores que murieron en 2004 por el HSM, 47\% fueron mujeres (mientras que $26 \%$ fueron hombres, y $28 \%$, niños de ambos sexos). ${ }^{1}$

Una buena noticia, dice Wipfli, es que el porcentaje de mujeres fumadoras en China ha permanecido bajo, pese a la preocupación de la comunidad de salud pública en el sentido de que la industria del tabaco pudiera buscar un mercado entre las mujeres: tradicionalmente las mujeres allí no han sido fumadoras, y esto es algo que la creciente riqueza y el creciente comercialismo podría haber cambiado. No obstante, en Europa y Asia, y en particular en los países de bajos ingresos situados en esas regiones y en los países donde casi todos los padres de familia fuman en casa, los niveles de exposición al HSM entre los no fumadores generalmente son sumamente elevados, dice Wipfli. Ella y Samet instan a que se implementen plenamente los diversos componentes del Convenio Marco de la OMS para el Control del Tabaquismo de la OMS (un tratado internacional que opera tanto sobre el abasto como sobre la demanda de tabaco), así como políticas y programas educativos relacionados.

La OMS presentará el año próximo un reporte sobre el número de naciones que han establecido la prohibición de fumar en los espacios públicos, incluyendo los lugares de trabajo y restaurantes, señala Armando Peruga, director del programa de la Iniciativa por un Mundo Libre de Tabaco de la OMS y coautor del reporte publicado en The Lancet. ${ }^{1}$ Peruga dice que el equipo necesita trabajar más para refinar sus cálculos y reunir más datos sobre los países individuales, en particular sobre aquellos para los cuales no se cuenta en absoluto con datos reportados sobre el tabaquismo; espera que el equipo pueda completar estos cálculos en un año más o menos.

Mientras tanto, el nuevo estudio es "un impresionante esfuerzo por producir un cálculo de los efectos globales del humo de segunda mano", dice Bart Ostro, científico investigador del Centro de Investigación en Epidemiología Ambiental (CREAL) de Barcelona, en licencia temporal de la Agencia de Protección al Medio Ambiente de California. Ostro 
comenta que las conclusiones del equipo están basadas en el empleo de un método epidemiológico bien establecido y que se "utilizaron muchos estudios que han sido objeto de numerosas revisiones por pares en el pasado", incluyendo el reporte de 2006 de la Inspectoría General de Salud (Surgeon General) de Estados Unidos, que tuvo gran influencia, y un reporte similar de la Agencia de Protección al Medio Ambiente de California publicado en 2005. El análisis de sensibilidad realizado por los investigadores "demuestra que, no importa qué supuestos se utilicen, el impacto sobre niños y adultos sigue siendo de gran importancia para la salud pública", añade Ostro.

Pese a las lagunas en los datos, el número estimado es "una cifra relevante para las políticas y que debiera motivar a la acción", dice Samet. "El propósito de estos ejercicios es proporcionar una orientación general y una comprensión de la magnitud de la carga de enfermedad... y de en qué medida podría evitarse mediante estrategias preventivas."

Naomi Lubick

es una escritora científica independiente que reside en Estocolmo, Suecia, y en

Folsom, CA. Ha escrito para las revistas Environmental Science \& Technology, Nature y Earth

\section{Referencias y notas}

I. Öberg M, et al. Worldwide burden of disease from exposure to second-hand smoke: a retrospective analysis of data from 192 countries. Lancet 377(9760):139-146 (201 I); doi:10.1016/ S0I40-6736(10)6/388-8.

2. DHHS. The health consequences of involuntary exposure to tobacco smoke.A report of the Surgeon General.Atlanta, GA:US Centers for Disease Control and Prevention, Public Health Service, US Department of Health and Human Services (2006). Disponible en: http://tinyurl.com/ o9d8r [consultado el 6 de enero de $201 \mathrm{I}$ ].
3. CDC/WHO. GlobalYouth Tobacco Survey (GYTS).Atlanta, GA:US Centers for Disease Control and Prevention; Geneva, Switzerland:World Health Organization. Disponible en: http://tinyurl. com/27qvy2q [consultado el 6 de enero de 201 I].

4. El informe de 2006 de la Inspectoría General de Salud de EU concluyó que la literatura cientíica no había podido establecer hasta el momento una conexión firme entre el cáncer de mama y el humo de segunda mano. Sin embargo, en una evaluación sobre efectos de salud en apoyo de una propuesta para identificar el HSM como un contaminante tóxico ambiental, la Agencia de Protección Ambiental de California concluyó que el peso de la evidencia soportaba una relación causal entre la exposición al HSM y el cáncer de mama en mujeres jóvenes (State of California Air Resources Board.Appendix III. Proposed Identification of Environmental Tobacco Smoke as a Toxic Air Contaminant. Part B-Health Effects. Sacramento, CA:California Environmental Protection Agency [2005].) Disponible en: http:// tinyurl.com/2wdv2mp [consultado el 6 de enero de 20l I]).

5. Wipfli HL, Samet JM. Second-hand smoke's worldwide disease toll. Lancet 377(9760): 10II02 (20I I); doi:I0.10I6/S0I40- 6736(I0)61922-8. 